\title{
Open scientist in the wonderland: advocating for blockchain-based decentralized applications for science*
}

\author{
Alcemir Rodrigues Santos ${ }^{1}$ \\ ${ }^{1}$ Universidade Estadual do Piauí (UESPI) \\ Campus Piripiri \\ alcemiraprp.uespi.br
}

\begin{abstract}
For too long now, centralized publication governance, unrewarded efforts for reviewing and editing high quality research have been on the spot. In this vision paper, We promote the idea of shifting the research communities collaboration and knowledge sharing towards blockchain-based decentralized platforms. Such change have the power to fulfill several requirements for open science from the starting point.
\end{abstract}

\section{Introduction}

Modern software engineering along with other science communities have been learning and improving their research methods to produce sound evidence over the years. However, apart from digitization and all the brand new technologies developed during this time, the fact that we share the scientific knowledge through centralized Scientific Publications Systems (SPS) have not really changed. Such fact raises several concerns with regarding the openness of science among open science advocates, such as $(i)$ the lack of access to the knowledge produced by the research community and (i) the lack of reward for working in the editorial boards.

Mendez et al. elaborated on the key concepts (e.g. open access, open data, open source, preregistration of studies, open science badges, and open peer review), engagement, implementation of open science for software engineering [Mendez et al. 2020]. Several initiatives cover each of these aspects to some extent (e.g., Zenodo ${ }^{1}$, Open Science Framework ${ }^{2}, \operatorname{arXiv}^{3}$, Journal of Open Source Software ${ }^{4}$ ), but they are centralized applications. In 2008, such dependency of a central authority started to fall apart as Satoshi Nakamoto proposed the Bitcoin [Nakamoto 2008]. While economists diverge on its value as fiat money, its technology (i.e., blockchain ${ }^{5}$ ) have already changed peer-to-peer transactions forever. It paved the room for innovations like the decentralized applications (DApps) like those provided by several blockchain organizations (e.g., Ethereum, Cardano, and Internet Computer). Some available blockchain (i) aim at fully decentralized governance, (ii) provide scalability, and (iii) run at Web speed, in which an open science ecosystem can be built upon.

\footnotetext{
*Open peer review artifacts for this paper are available at https: / / zenodo.org/communities / opensciense 2021

${ }^{1}$ Details at: https://zenodo.org/

${ }^{2}$ Details at: https://osf.io/

${ }^{3}$ Details at: http://arxiv.org/

${ }^{4}$ Details at: https://joss.theoj.org/about

${ }^{5}$ The blockchain is a type of distributed database that stores public transactions in a chain of data organized in blocks of trusted information.
} 


\section{Blockchain and DApps for Open Science}

DApps (i.e., blockchain) are growing fast in number, size, and domains (e.g., finance, healthcare, education, logistics, real estate). It is possible for open science ecosystem to benefit too. Among several solutions that could be built as DApps, decentralized SPS are the means for research institutions to pay science and keep it at home. Next, we highlight both commercial and research proposals found.

Commercial approaches. "Blockchain for Science" ${ }^{6 "}$ is a think tank that wants to use blockchain technology for connecting all research devices and distributing research money anonymously creating a new living knowledge network and they are not alone. Initiatives such as the "Decentralized Science" "and "Orvium", "ARTiFACTS", and "Manubot ${ }^{10 "}$ [Himmelstein et al. 2019] are building decentralized SPS using blockchain technology. Such initiatives advocate towards rewarding faster and better peer-reviewing and incentives to the publication of negative results.

Research approaches. Mackey et al. proposed a governance framework based on a consortium blockchain model to create a more efficient means of navigating the publishing process [Mackey et al. 2019]. Highlights to EUREKA [Niya et al. 2019] and DASP [Evaristo et al. 2019], blockchain-based scientific publishing platforms built upon Ethereum network. Nugyen et al. [Nguyen et al. 2021] carried an qualitative study on how blockchain technology can be used to facilitate the social value creation of crowdfunding when adopted as an alternative infrastructure and demonstrated practical applications of blockchain use in crowdfunding.

\section{Challenges ahead}

Although blockchain is not mainstream yet, the huge efforts from the several nongovernmental organization foundations claims it will be in the near future. The amount of initiatives we presented show the research community is giving more and more attention to the benefits of using the technology. Next, we discuss the challenges ahead for the construction of truly open ecosystem.

Challenge 1 - To provide access to the produced knowledge (open data/access). With an SPS DApp, every research institution could run a blockchain node and have a copy of every paper ever published in the platform. The infrastructure is already there for most of them. For this matter, we need $(i)$ to build or develop the already existent open-source decentralized SPS; (ii) to increase the awareness of initiatives trying to replace the current SPS by the scientific community at large, as it is still timid and there is plenty of room for all of them to survive.

Challenge 2 - To reward for contribution and fund open research. Research DApps tokens can be monetized throughout the publication process and be used to reward researchers acting either as scientists, peer-reviewers, or editors. Moreover, these tokens could be used to help funding research proposals committed to open science principles preregistered in the platform.

\footnotetext{
${ }^{6}$ Available at https://www.blockchainforscience.com

${ }^{7}$ Details at https: / / decentralized.science/

${ }^{8}$ Details at https: / / orvium.io/

${ }^{9}$ Details at https: / / artifacts.ai/

${ }^{10}$ Details at https : / manubot.org/
} 
Challenge 3 - To distribute the governance. The research community should be in control of the publication process rather than business corporations, which could be achieved on a blockchain-based decentralized governance by researchers holding research tokens. A blockchain's token would allow researcher to decide on future of the publication process and the ecosystem's priorities.

\section{Road to the wonderland}

I believe the computer science and software engineering communities have opportunity to lead the change. In this sense, we enumerate next some research directions that can both guide open scientists to the wonderland and improve scientific community awareness DApps benefits as a side effect.

Research Direction 1. Specification and implementation of a open research DApps including SPS, and its tokenomics.

Research Direction 2. Tools, processes and strategies for modeling, development, verification, validation, and testing of smart contracts and DApps.

Research Direction 3. Integration between existing open systems as DApps and blockchain networks available.

Research Direction 4. Formal education on the implementation of blockchain development, smart contracts, DApps.

\section{References}

Evaristo, B., Nascimento, V., Defrémont, A., Pinheiro, B., and Abelém, A. (2019). Editora científica autônoma e distribuída sobre blockchain privada. In Anais do II Workshop em Blockchain: Teoria, Tecnologia e Aplicações, Porto Alegre, RS, Brasil. SBC.

Himmelstein, D. S., Rubinetti, V., Slochower, D. R., Hu, D., Malladi, V. S., Greene, C. S., and Gitter, A. (2019). Open collaborative writing with manubot. PLOS Computational Biology, 15:21.

Mackey, T. K., Shah, N., Miyachi, K., Short, J., and Clauson, K. (2019). A framework proposal for blockchain-based scientific publishing using shared governance. Frontiers in Blockchain, 2:19.

Mendez, D., Graziotin, D., Wagner, S., and Seibold, H. (2020). Open Science in Software Engineering, pages 477-501. Springer International Publishing, Cham.

Nakamoto, S. (2008). Bitcoin: A peer-to-peer electronic cash system. https: //bitcoin.org/bitcoin.pdf. Accessed: 2021-Jun-09.

Nguyen, L. T., Hoang, T. G., Do, L. H., Ngo, X. T., Nguyen, P. H., Nguyen, G. D., and Nguyen, G. N. (2021). The role of blockchain technology-based social crowdfunding in advancing social value creation. Technological Forecasting and Social Change, 170:120898.

Niya, S. R., Pelloni, L., Wullschleger, S., Schaufelbühl, A., Bocek, T., Rajendran, L., and Stiller, B. (2019). A blockchain-based scientific publishing platform. In IEEE International Conference on Blockchain and Cryptocurrency, ICBC Proceedings, pages 329-336. IEEE. 